\title{
Oscillator Strengths for Allowed Transitions in Li(II)
}

\author{
Ş. ATEŞ* AND G. ÇELIK \\ Department of Physics, Faculty of Science, Selçuk University, Campus 42049 Konya, Turkey
}

(Received March 6, 2009; in final form May 14, 2009)

\begin{abstract}
Electric dipole oscillator strengths have been computed for transitions between both multiplet and individual lines in the $\mathrm{Li}(\mathrm{II})$ ion. The weakest bound electron potential model theory has been used. We have employed both numerical Coulomb approximation wave functions and numerical non-relativistic Hartree-Fock wave functions in the determination of expectation values of radii. The necessary energy values have been taken from experimental ionization energies. The oscillator strengths calculated with parameters obtained by using the two different wave functions have been compared not only to each other but also to other data taken from literature. A good agreement with results in literature has been obtained.
\end{abstract}

PACS numbers: 31.15.ag, 32.70.Cs

\section{Introduction}

Some spectroscopic data such as oscillator strengths and transition probabilities are required in laser spectroscopy, for new atomic clocks, laboratory plasma diagnostic, astrophysical studies, studies of controlled thermonuclear reactions and Tokamak devices. Therefore, the accurate estimate of these data in the atomic and ionic systems is important. The calculation of some physical parameters corresponding to individual energy levels especially to highly lying levels and Rydberg states in multi-electron atoms is always a difficult problem in theoretical studies. Many methods such as the HartreeFock approximations, configuration interaction methods, semi-empirical methods and many-body perturbation theories exist for the calculation of transition probabilities, oscillator strengths and lifetimes of excited levels for atomic or ionic systems. The lithium is an important test case for atomic theory. The considered approximations in atomic structure theory must be capable first to represent accurately lithium atom in order to have possibility to calculate reasonably the multi-electron atoms. Theoretical calculations in atomic lithium have been widely studied by many authors [1-12]. The most sensitive results have been presented by Yan and Drake [4], McAlexander et al. [5] and Zhang et al. [11]. Yan and Drake have calculated the non-relativistic oscillator strengths for the $1 s^{2} 2 s$ ${ }^{2} S-1 s^{2} 2 p{ }^{2} P^{\mathrm{o}}$ and $1 s^{2} 2 p{ }^{2} P^{\mathrm{o}}-1 s^{2} 3 d{ }^{2} D$ multiplet transitions with an accuracy of $\pm 6 \times 10^{-6}$ using variational wave functions in the Hylleraas coordinates. McAlexander et al. have extracted a value for $2 p^{2} P^{\circ}$ lifetime via photoassociation. Zhang et al. have determined oscillator

* corresponding author; e-mail: suleates@selcuk.edu.tr strengths and dispersion coefficients of a number of the low-lying states of lithium. They have calculated wave functions and energies in frozen core approximation. Although ionized lithium is the second member of the helium isoelectronic sequence, $\operatorname{Li}(\mathrm{II})$ has not been extensively studied experimentally. Theoretically, Li(II) has been extensively treated as a member of the He isoelectronic sequence. The theoretical calculation of oscillator strengths is usually a difficult task. The calculations include Hylleraas-type wave functions. Schiff et al. have calculated oscillator strengths for members of the helium isoelectronic sequence up to $Z=10$ [13]. Drake has presented oscillator strengths for some dominant transitions of helium and helium-like ions using the HylleraasScherr-Knight variation-perturbation method [14]. Anderson and Weinhold have presented dipole oscillator strengths of the low-lying singlet and triplet transitions in $\mathrm{He}$ and $\mathrm{Li}(\mathrm{II})$ using Hylleraas-type wave functions [15]. Kono and Hattori have calculated nonrelativistic oscillator strengths for the transitions $n \leq 5$ in helium-like ions with $Z=3-7$ [16]. Theodosiou has calculated the Rydberg state lifetimes and oscillator strengths for the $s-p$ and $p-d$ transitions in the singly ionized lithium using Hylleraas-type wave functions [17].

The data in the literature include generally the Coulomb approximation results for $\mathrm{Li}(\mathrm{II})$. This approximation is valid in case of large radial distances of active electron. The weakest bound electron potential model (WBEPM) theory presents reliable results for both large and small radial distances of active electron. In the present work, many transitions have been considered in order to show validity of the WBEPM theory. The absorption oscillator strengths have been calculated in $\mathrm{Li}(\mathrm{II})$ ion. The obtained results have been compared with available data in literature. 


\section{Theoretical procedure}

The absorption oscillator strength or $f$-value for an electric dipole transition between an initial state $|j\rangle$ and a final state $\left|j^{\prime}\right\rangle$ is given as [18]:

$$
f_{j j^{\prime}}=\frac{2\left(E_{J^{\prime}}-E_{J}\right)}{3(2 J+1)} S .
$$

Here, $E_{J^{\prime}}-E_{J}$ is the transition energy in atomic units, $2 J+1$ is the degeneracy of initial level and $S$ is the electric dipole line strength in atomic units. Line strength is determined according to the coupling schemes and the transition types in atomic or ionic systems. The text book written by Cowan [18] reports in detail, how the line strength can be calculated according to coupling schemes being considered and for different transition types. The most important quantity for the calculation of $S$ line strength is determination of the radial transition integral or transition matrix element. $L S$ coupling is the dominant coupling scheme in light atoms and electric dipole line strength for transitions between two excited levels in this coupling scheme can be given to be [18]:

$$
\begin{aligned}
& \sqrt{S_{L S}} \equiv\left\langle\left[\left(\ldots \alpha_{1} L_{1}, l_{2}\right) L\left(\ldots S_{1} s_{2}\right) S\right] J|| \boldsymbol{r}_{N}^{(1)}\right| \\
& \times\left|\left[\left(\ldots \alpha_{1}^{\prime} L_{1}^{\prime}, l_{2}^{\prime}\right) L^{\prime}\left(\ldots S_{1}^{\prime} s_{2}\right) S^{\prime}\right] J^{\prime}\right\rangle \\
& =(-1)^{S+J^{\prime}+L_{1}+l_{2}^{\prime}}\left[J, J^{\prime}, L, L^{\prime}\right]^{1 / 2} \\
& \quad \times\left\{\begin{array}{ccc}
L & S & J \\
J^{\prime} & 1 & L^{\prime}
\end{array}\right\}\left\{\begin{array}{ccc}
L_{1} & l_{2} & L \\
1 & L^{\prime} & l_{2}^{\prime}
\end{array}\right\} P_{l_{2} l_{2^{\prime}}}^{(1)}, \\
& \left\{\begin{array}{ccc}
L & S & J \\
J^{\prime} & 1 & L^{\prime}
\end{array}\right\}=W(a b c d ; e f), \\
& P_{l_{i} l_{f}}^{(1)}=l_{>}\left\langle n_{i}, l_{i}\left|r^{k}\right| n_{f}, l_{f}\right\rangle \\
& =l_{>} \int_{0}^{\infty} r^{k+2} R_{n_{i} l_{i}}(r) R_{n_{f} l_{f}}(r) \mathrm{d} r .
\end{aligned}
$$

If there are $n$ equivalent electrons in a shell, the expression for line strength should be multiplied by the factor of $n\left(l_{1}^{n} \alpha_{1} L_{1} S_{1}\left\{\mid l_{1}^{n-1} \alpha_{1}^{\prime} L_{1}^{\prime} S_{1}^{\prime}\right)\right.$ in transitions including equivalent electrons

$$
\begin{aligned}
& \left.\sqrt{S_{L S}} \equiv\left\langle\alpha_{1} L_{1}, S_{1}, J|| \boldsymbol{r}^{(1)}|| \alpha_{1}^{\prime} L_{1}^{\prime}, S_{1}^{\prime}, l_{2}\right) L^{\prime} S^{\prime} J^{\prime}\right\rangle \\
& =\delta_{S_{1} S^{\prime}}(-1)^{L_{1}^{\prime}+l_{2}+S_{1}+J^{\prime}}\left[n\left(L_{1}, L^{\prime}, J, J^{\prime}\right)\right]^{1 / 2} \\
& \times\left\{\begin{array}{ccc}
L_{1} & S & J \\
J^{\prime} & 1 & L^{\prime}
\end{array}\right\}\left\{\begin{array}{ccc}
l_{1} & L_{1}^{\prime} & L_{1} \\
L^{\prime} & 1 & l_{2}
\end{array}\right\} \\
& \times\left(l_{1}^{n} \alpha_{1} L_{1} S_{1}\left\{\mid l_{1}^{n-1} \alpha_{1}^{\prime} L_{1}^{\prime} S_{1}^{\prime}\right) P_{l_{2} l_{2^{\prime}}}^{(1)} .\right.
\end{aligned}
$$

Here, $n$ is the number of equivalent electrons in a shell and the bracketed term is the coefficient of fractional parentage. The tables of numerical values of the fractional parentage coefficients are given in the literature for various shells $[18,19]$. On the other hand, $W(a b c d ; e f)$ is called as the Racah coefficient or Wigner's 6- $j$ symbol and it is used to describe the coupling between two or more angular momentum. $P_{l_{2} l_{2^{\prime}}}^{(1)}$ quantity given in Eq. (3) is known to be radial transition integral or transition matrix element.

In this work, the WBEPM theory has been employed to calculate the radial transition integral. This theory is based on distinction of the weakest bound electrons (WBE) and non-weakest bound electrons (NWBE) in given atomic or ionic systems. By the separation of the electrons in a given system, complex many-electron problem can be simplified as the single electron problem and so can be solved easily. This theory has been developed by Zheng [20, 21] and has been applied to calculate various atomic properties such as energy levels, ionization potentials, transition probabilities, oscillator strengths and lifetimes of excited levels in the many-electron atomic and ionic systems [22-26]. In the WBEPM theory, electronic radial wave functions are presented as a function of the Laguerre polynomial in terms of some parameters which are determined using the experimental energy data and the expectation values of radii $[22-26]$,

$$
\begin{aligned}
& R_{n l}(r)=\frac{\left(\frac{2 Z^{*}}{n^{*}}\right)^{l^{*}+3 / 2}}{\left[\frac{2 n^{*}}{(n-l-1) !} \Gamma\left(n^{*}+l^{*}+1\right)\right]^{1 / 2}} \\
& \quad \times \exp \left(-\frac{Z^{*} r}{n^{*}}\right) r^{l^{*}} L_{n-l-1}^{2 l^{*}+1}\left(\frac{2 Z^{*} r}{n^{*}}\right) .
\end{aligned}
$$

After obtaining the relevant parameters, radial transition integral or radial matrix element between two different states can be determined easily using radial wave functions given in Eq. (4). The expectation value of $r^{k}$ or radial transition integral for $k=1$ in case of transition from the level $\left(n_{i}, l_{i}\right)$ to the level $\left(n_{f}, l_{f}\right)$ can be obtained as $[22,23]$ :

$$
\begin{aligned}
& \left\langle n_{i}, l_{i}\left|r^{k}\right| n_{f}, l_{f}\right\rangle=\int_{0}^{\infty} r^{k+2} R_{n_{i} l_{i}}(r) R_{n_{f} l_{f}}(r) \mathrm{d} r \\
& =(-1)^{n_{f}+n_{i}+l_{f}+l_{i}}\left(\frac{2 Z_{f}^{*}}{n_{f}^{*}}\right)^{l_{f}^{*}}\left(\frac{2 Z_{i}^{*}}{n_{i}^{*}}\right)^{l_{i}^{*}} \\
& \times\left(\frac{Z_{f}^{*}}{n_{f}^{*}}-\frac{Z_{i}^{*}}{n_{i}^{*}}\right)^{-l_{f}^{*}-l_{i}^{*}-k-3}\left[\frac{n_{f}^{*^{4}} \Gamma\left(n_{f}^{*}+l_{f}^{*}+1\right)}{4 Z_{f}^{*^{3}}\left(n_{f}-l_{f}-1\right) !}\right]^{-1 / 2} \\
& \quad \times\left[\frac{n_{i}^{*^{4}} \Gamma\left(n_{i}^{*}+l_{i}^{*}+1\right)}{4 Z_{i}^{*^{3}}\left(n_{i}-l_{i}-1\right) !}\right]^{-1 / 2} \sum_{n_{1}-l_{f}-1}^{n_{m_{i}-l_{i}-1}} \sum_{m_{2}=0}^{(-1)^{m_{2}}} \frac{m_{1} ! m_{2} !}{}
\end{aligned}
$$




$$
\begin{aligned}
& \times\left(\frac{Z_{f}^{*}}{n_{f}^{*}}-\frac{Z_{i}^{*}}{n_{i}^{*}}\right)^{m_{1}+m_{2}}\left(\frac{Z_{f}^{*}}{n_{f}^{*}}+\frac{Z_{i}^{*}}{n_{i}^{*}}\right)^{-m_{1}-m_{2}} \\
& \times \Gamma\left(l_{f}^{*}+l_{i}^{*}+m_{1}+m_{2}+k+3\right) \\
& \times \sum_{m_{3}=0}^{S}\left(\begin{array}{l}
l_{i}^{*}-l_{f}^{*}+k+m_{2}+1 \\
n_{f}^{*}-l_{f}^{*}-1-m_{1}-m_{3}
\end{array}\right) \\
& \times\left(\begin{array}{c}
l_{f}^{*}-l_{i}^{*}+k+m_{1}+1 \\
n_{i}^{*}-l_{i}^{*}-1-m_{2}-m_{3}
\end{array}\right) \\
& \times\left(\begin{array}{c}
l_{i}^{*}+l_{f}^{*}+k+m_{1}+m_{2}+m_{3}+2 \\
m_{3}
\end{array}\right) .
\end{aligned}
$$

In order to solve this radial transition integral, $Z^{*}, n^{*}$ and $l^{*}$ parameters must be known. These parameters are obtained by solving the following couple of equations:

$$
\begin{aligned}
& I=-\varepsilon=\frac{Z^{*^{2}}}{2 n^{*^{2}}}, \\
& \langle r\rangle=\frac{3 n^{*^{2}}-l^{*}\left(l^{*}+1\right)}{2 Z^{*}} .
\end{aligned}
$$

Here $I$ is ionization energy and $\langle r\rangle$ is expectation value for radius of weakest bound electron. In the determination of $Z^{*}, n^{*}$ and $l^{*}$ parameters, relevant energy values have been taken from experimental energy data in the literature and expectation values for the radii of levels have been calculated by using wave functions obtained from two different theoretical approximations. The papers given by Zheng et al. describe in detail the WBEPM theory [20-26].

\section{Results and conclusion}

In the present paper, the electric dipole oscillator strengths have been computed using the WBEPM theory in $\mathrm{Li}(\mathrm{II})$ ion. The experimental energy values and expectation values of radii belonging to states are used in Eqs. (6) and (7) to obtain the $Z^{*}, n^{*}$ and $l^{*}$ parameters. The expectation values of radii for all of states have been determined by using both numerical Coulomb approximation (NCA) wave functions [27] and non-relativistic Hartree-Fock (NRHF) wave functions [28]. The necessary energy values have been taken from experimental energy data in the literature [29]. The obtained parameters are used in Eqs. (5), (2) and (1) to yield oscillator strengths between whole states. The WBEPM theory is a semi-empirical method. It is a one-electron approximation which assumes that each radiative transition rate can be approximated by hydrogenic expressions in which the orbital parameters are fitted to both energy and radius of the weakest bound electron. In this method, relativistic effects are neglected except for the binding energies. The expectation values of radii and energy values which are used in determination of $Z^{*}, n^{*}$ and $l^{*}$ parameters are very important. The determination of the expectation values of radii is more crucial than energy values in the WBEPM theory, because experimental ionization energy data are very precise. In this study, we have employed both NCA wave functions and NRHF wave functions in determination of relevant parameters to obtain more sensitive and reliable results. This study presents a comparison of oscillator strengths calculated by using different parameters obtained from two approximation wave functions in the WBEPM theory framework for $\mathrm{Li}(\mathrm{II})$ ion.

TABLE I

Multiplet oscillator strengths for some lines of atomic lithium.

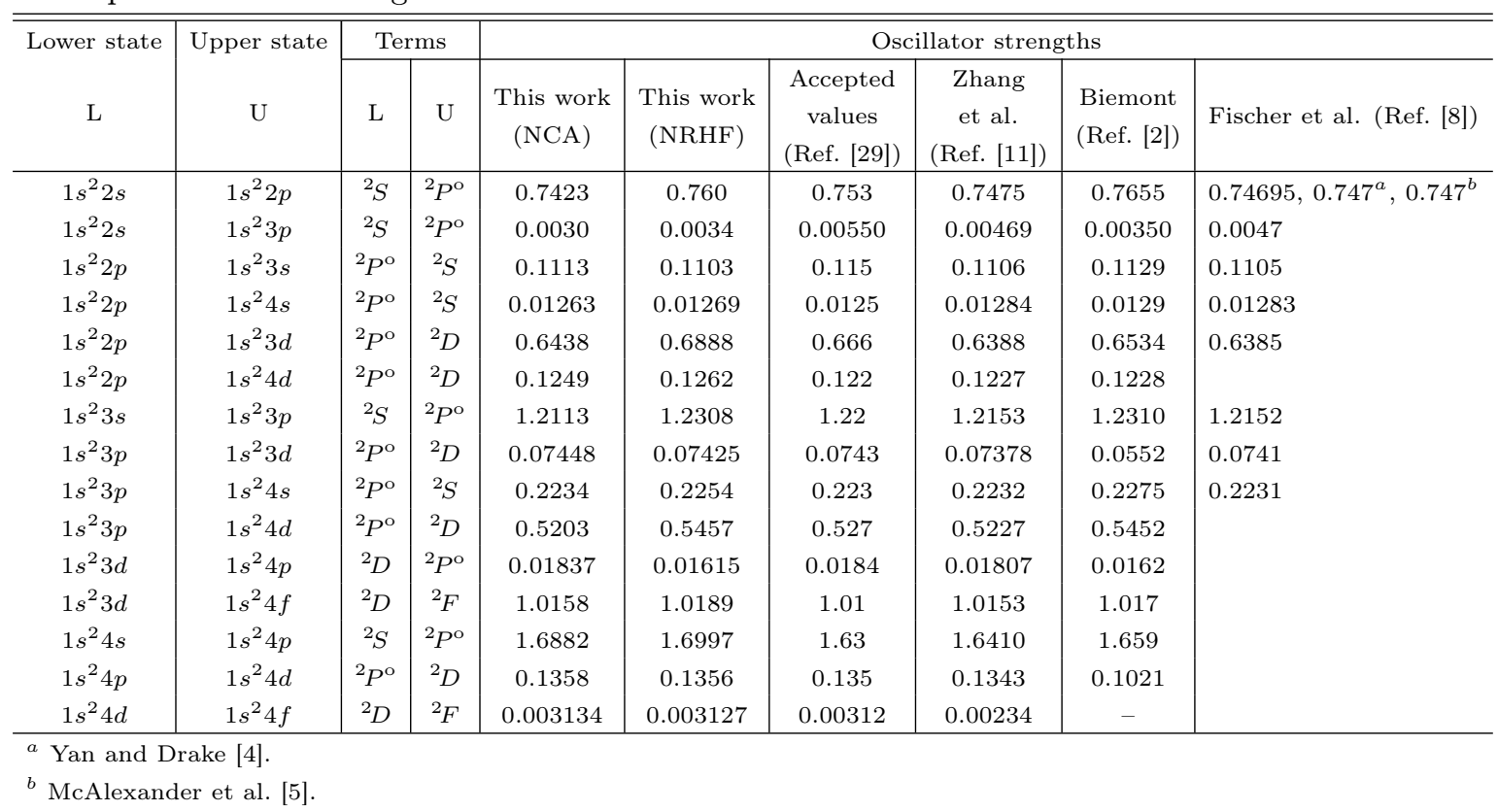


TABLE II

Absorption oscillator strengths for $\mathrm{Li}(\mathrm{II})$.

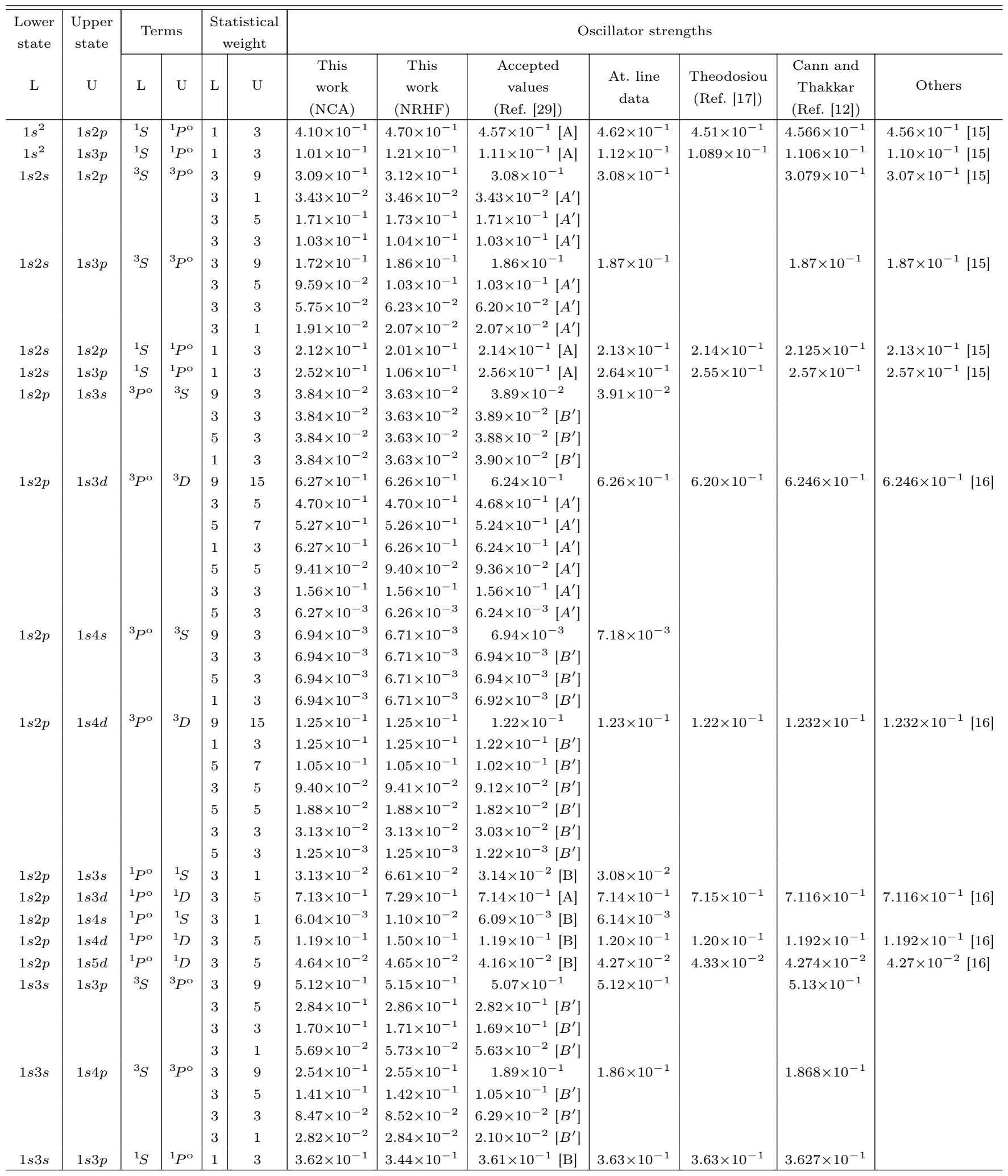


Absorption oscillator strengths for $\operatorname{Li}(\mathrm{II})$.

TABLE II (cont.)

\begin{tabular}{|c|c|c|c|c|c|c|c|c|c|c|c|c|}
\hline \multirow{2}{*}{$\begin{array}{c}\text { Lower } \\
\text { state } \\
\text { L }\end{array}$} & \multirow{2}{*}{$\begin{array}{c}\text { Upper } \\
\text { state } \\
\text { U }\end{array}$} & \multicolumn{2}{|c|}{ Terms } & \multicolumn{2}{|c|}{$\begin{array}{c}\text { Statistical } \\
\text { weight }\end{array}$} & \multicolumn{7}{|c|}{ Oscillator strengths } \\
\hline & & $\mathrm{L}$ & $\mathrm{U}$ & $\mathrm{L}$ & $\mathrm{U}$ & $\begin{array}{l}\text { This work } \\
\text { (NCA) }\end{array}$ & $\begin{array}{l}\text { This work } \\
\text { (NRHF) }\end{array}$ & $\begin{array}{c}\text { Accepted } \\
\text { values } \\
\text { (Ref. [29]) }\end{array}$ & $\begin{array}{l}\text { At. line } \\
\text { data }\end{array}$ & $\begin{array}{c}\text { Theodosiou } \\
\text { (Ref. [17]) }\end{array}$ & $\begin{array}{l}\text { Cann and } \\
\text { Thakkar } \\
\text { (Ref. [12]) }\end{array}$ & $\begin{array}{c}\text { Kono and } \\
\text { Hattori } \\
\text { (Ref. [16]) }\end{array}$ \\
\hline \multirow[t]{7}{*}{$1 s 3 p$} & \multirow[t]{7}{*}{$1 s 3 d$} & \multirow[t]{7}{*}{${ }^{3} P^{\circ}$} & \multirow[t]{7}{*}{${ }^{3} D$} & 9 & 15 & $9.08 \times 10^{-2}$ & $9.08 \times 10^{-2}$ & $9.05 \times 10^{-2}$ & \multirow[t]{7}{*}{$9.08 \times 10^{-2}$} & \multirow[t]{11}{*}{$9.05 \times 10^{-2}$} & \multirow[t]{11}{*}{$9.08 \times 10^{-2}$} & \multirow[t]{11}{*}{$9.07 \times 10^{-2}$} \\
\hline & & & & 5 & 7 & $7.63 \times 10^{-2}$ & $7.62 \times 10^{-2}$ & $7.61 \times 10^{-2}\left[A^{\prime}\right]$ & & & & \\
\hline & & & & 3 & 5 & $6.81 \times 10^{-2}$ & $6.81 \times 10^{-2}$ & $6.79 \times 10^{-2}\left[A^{\prime}\right]$ & & & & \\
\hline & & & & 1 & 3 & $9.08 \times 10^{-2}$ & $9.07 \times 10^{-2}$ & $9.06 \times 10^{-2}\left[A^{\prime}\right]$ & & & & \\
\hline & & & & 5 & 5 & $1.36 \times 10^{-2}$ & $1.36 \times 10^{-2}$ & $1.36 \times 10^{-2}\left[A^{\prime}\right]$ & & & & \\
\hline & & & & 3 & 3 & $2.27 \times 10^{-2}$ & $2.27 \times 10^{-2}$ & $2.26 \times 10^{-2}\left[A^{\prime}\right]$ & & & & \\
\hline & & & & 5 & 3 & $9.08 \times 10^{-4}$ & $9.08 \times 10^{-4}$ & $9.06 \times 10^{-4}\left[A^{\prime}\right]$ & & & & \\
\hline \multirow[t]{4}{*}{$1 s 3 p$} & \multirow[t]{4}{*}{$1 s 4 s$} & \multirow[t]{4}{*}{${ }^{3} P^{\mathrm{o}}$} & ${ }^{3} S$ & 9 & 3 & $8.41 \times 10^{-2}$ & $8.40 \times 10^{-2}$ & $8.50 \times 10^{-2}$ & $8.51 \times 10^{-2}$ & & & \\
\hline & & & & 3 & 3 & $8.41 \times 10^{-2}$ & $8.40 \times 10^{-2}$ & $8.51 \times 10^{-2}\left[B^{\prime}\right]$ & & & & \\
\hline & & & & 5 & 3 & $8.41 \times 10^{-2}$ & $8.40 \times 10^{-2}$ & $8.51 \times 10^{-2}\left[B^{\prime}\right]$ & & & & \\
\hline & & & & 1 & 3 & $8.41 \times 10^{-2}$ & $8.40 \times 10^{-2}$ & $8.50 \times 10^{-2}\left[B^{\prime}\right]$ & & & & \\
\hline $1 s 3 p$ & $1 s 4 d$ & ${ }^{3} P^{\circ}$ & ${ }^{3} D$ & 9 & 15 & $5.00 \times 10^{-1}$ & $5.10 \times 10^{-1}$ & $5.09 \times 10^{-1}$ & $5.05 \times 10^{-1}$ & $5.02 \times 10^{-1}$ & $5.033 \times 10^{-1}$ & $5.034 \times 10^{-1}$ \\
\hline & & & & 3 & 3 & $1.25 \times 10^{-1}$ & $1.27 \times 10^{-1}$ & $1.27 \times 10^{-1}\left[B^{\prime}\right]$ & & & & \\
\hline & & & & 5 & 7 & $4.20 \times 10^{-1}$ & $4.28 \times 10^{-1}$ & $4.28 \times 10^{-1}\left[B^{\prime}\right]$ & & & & \\
\hline & & & & 5 & 5 & $7.50 \times 10^{-2}$ & $7.65 \times 10^{-2}$ & $7.66 \times 10^{-2}\left[B^{\prime}\right]$ & & & & \\
\hline & & & & 5 & 3 & $5.00 \times 10^{-3}$ & $5.10 \times 10^{-3}$ & $5.10 \times 10^{-3}\left[B^{\prime}\right]$ & & & & \\
\hline & & & & 3 & 5 & $3.75 \times 10^{-1}$ & $3.82 \times 10^{-1}$ & $3.83 \times 10^{-1}\left[B^{\prime}\right]$ & & & & \\
\hline & & & & 1 & 3 & $5.00 \times 10^{-1}$ & $5.10 \times 10^{-1}$ & $5.10 \times 10^{-1}\left[B^{\prime}\right]$ & & & & \\
\hline $1 s 3 p$ & $1 s 5 s$ & ${ }^{3} P^{\mathrm{o}}$ & ${ }^{3} S$ & 9 & 3 & $1.52 \times 10^{-2}$ & $1.53 \times 10^{-2}$ & $1.58 \times 10^{-2}$ & $1.60 \times 10^{-2}$ & & & \\
\hline & & & & 3 & 3 & $1.52 \times 10^{-2}$ & $1.53 \times 10^{-2}$ & $1.58 \times 10^{-2}\left[B^{\prime}\right]$ & & & & \\
\hline & & & & 5 & 3 & $1.52 \times 10^{-2}$ & $1.53 \times 10^{-2}$ & $1.59 \times 10^{-2}\left[B^{\prime}\right]$ & & & & \\
\hline & & & & 1 & 3 & $1.52 \times 10^{-2}$ & $1.53 \times 10^{-2}$ & $1.58 \times 10^{-2}\left[B^{\prime}\right]$ & & & & \\
\hline $1 s 3 p$ & $1 s 6 d$ & ${ }^{3} P^{\circ}$ & ${ }^{3} D$ & 9 & 15 & $5.24 \times 10^{-2}$ & $5.33 \times 10^{-2}$ & $5.29 \times 10^{-2}$ & $5.39 \times 10^{-2}$ & & $5.388 \times 10^{-2}$ & \\
\hline & & & & 3 & 3 & $1.31 \times 10^{-2}$ & $1.33 \times 10^{-2}$ & $1.32 \times 10^{-2}\left[B^{\prime}\right]$ & & & & \\
\hline & & & & 5 & 7 & $4.40 \times 10^{-2}$ & $4.47 \times 10^{-2}$ & $4.45 \times 10^{-2}\left[B^{\prime}\right]$ & & & & \\
\hline & & & & 3 & 5 & $3.93 \times 10^{-2}$ & $3.99 \times 10^{-2}$ & $3.97 \times 10^{-2}\left[B^{\prime}\right]$ & & & & \\
\hline & & & & 1 & 3 & $5.24 \times 10^{-2}$ & $5.33 \times 10^{-2}$ & $5.28 \times 10^{-2}\left[B^{\prime}\right]$ & & & & \\
\hline & & & & 5 & 5 & $7.87 \times 10^{-3}$ & $7.99 \times 10^{-3}$ & $7.94 \times 10^{-3}\left[B^{\prime}\right]$ & & & & \\
\hline & & & & 5 & 3 & $5.24 \times 10^{-4}$ & $5.33 \times 10^{-4}$ & $5.30 \times 10^{-4}\left[B^{\prime}\right]$ & & & & \\
\hline $1 s 3 d$ & $1 s 4 p$ & ${ }^{3} D$ & ${ }^{3} P^{\circ}$ & 15 & 9 & $1.99 \times 10^{-2}$ & $1.89 \times 10^{-2}$ & $1.99 \times 10^{-2}$ & $1.96 \times 10^{-2}$ & & & \\
\hline & & & & 7 & 5 & $1.99 \times 10^{-2}$ & $1.89 \times 10^{-2}$ & $1.99 \times 10^{-2}\left[B^{\prime}\right]$ & & & & \\
\hline & & & & 5 & 3 & $1.49 \times 10^{-2}$ & $1.41 \times 10^{-2}$ & $1.49 \times 10^{-2}\left[B^{\prime}\right]$ & & & & \\
\hline & & & & 3 & 1 & $1.10 \times 10^{-2}$ & $1.05 \times 10^{-2}$ & $1.10 \times 10^{-2}\left[B^{\prime}\right]$ & & & & \\
\hline & & & & 5 & 5 & $4.97 \times 10^{-3}$ & $4.73 \times 10^{-3}$ & $4.96 \times 10^{-3}\left[B^{\prime}\right]$ & & & & \\
\hline & & & & 3 & 3 & $8.30 \times 10^{-3}$ & $7.88 \times 10^{-3}$ & $8.27 \times 10^{-3}\left[B^{\prime}\right]$ & & & & \\
\hline & & & & 3 & 5 & $5.53 \times 10^{-4}$ & $5.25 \times 10^{-4}$ & $5.52 \times 10^{-4}\left[B^{\prime}\right]$ & & & & \\
\hline $1 s 3 d$ & $1 s 5 p$ & ${ }^{3} D$ & ${ }^{3} P^{\circ}$ & 15 & 9 & $3.66 \times 10^{-3}$ & $3.47 \times 10^{-3}$ & $3.68 \times 10^{-3}$ & $3.71 \times 10^{-3}$ & & & \\
\hline & & & & 7 & 5 & $3.66 \times 10^{-3}$ & $3.47 \times 10^{-3}$ & $3.68 \times 10^{-3}\left[B^{\prime}\right]$ & & & & \\
\hline & & & & 5 & 3 & $2.74 \times 10^{-3}$ & $2.60 \times 10^{-3}$ & $2.76 \times 10^{-3}\left[B^{\prime}\right]$ & & & & \\
\hline & & & & 3 & 1 & $2.03 \times 10^{-3}$ & $1.92 \times 10^{-3}$ & $2.05 \times 10^{-3}\left[B^{\prime}\right]$ & & & & \\
\hline & & & & 5 & 5 & $9.14 \times 10^{-4}$ & $8.68 \times 10^{-4}$ & $9.22 \times 10^{-4}\left[B^{\prime}\right]$ & & & & \\
\hline & & & & 3 & 3 & $1.52 \times 10^{-3}$ & $1.44 \times 10^{-3}$ & $1.54 \times 10^{-3}\left[B^{\prime}\right]$ & & & & \\
\hline & & & & 3 & 5 & $1.01 \times 10^{-4}$ & $9.65 \times 10^{-5}$ & $1.02 \times 10^{-4}\left[B^{\prime}\right]$ & & & & \\
\hline $1 s 3 d$ & $1 s 3 p$ & ${ }^{1} D$ & ${ }^{1} P^{\circ}$ & 5 & 3 & $1.45 \times 10^{-2}$ & $1.45 \times 10^{-2}$ & $1.56 \times 10^{-2}[B]$ & $1.46 \times 10^{-2}$ & & & \\
\hline $1 s 3 d$ & $1 s 4 p$ & ${ }^{1} D$ & ${ }^{1} P^{\circ}$ & 5 & 3 & $8.97 \times 10^{-3}$ & $8.20 \times 10^{-3}$ & $9.11 \times 10^{-3}[B]$ & $8.90 \times 10^{-3}$ & & & \\
\hline $1 s 3 p$ & $1 s 4 s$ & ${ }^{1} P^{\mathrm{o}}$ & ${ }^{1} S$ & 3 & 1 & $6.87 \times 10^{-2}$ & $1.31 \times 10^{-1}$ & $6.86 \times 10^{-2}[B]$ & $6.69 \times 10^{-2}$ & & & \\
\hline $1 s 3 p$ & $1 s 4 d$ & ${ }^{1} P^{\mathrm{o}}$ & ${ }^{1} D$ & 3 & 5 & $6.52 \times 10^{-1}$ & $6.68 \times 10^{-1}$ & $6.53 \times 10^{-1}[B]$ & $6.54 \times 10^{-1}$ & & $6.5174 \times 10^{-1}$ & $6.517 \times 10^{-1}$ \\
\hline $1 s 3 p$ & $1 s 5 s$ & ${ }^{1} P^{\mathrm{o}}$ & ${ }^{1} S$ & 3 & 1 & $1.32 \times 10^{-2}$ & $2.28 \times 10^{-2}$ & $1.38 \times 10^{-2}[B]$ & $1.36 \times 10^{-2}$ & & & \\
\hline $1 s 3 p$ & $1 s 5 d$ & ${ }^{1} P^{\mathrm{o}}$ & ${ }^{1} D$ & 3 & 5 & $1.30 \times 10^{-1}$ & $1.33 \times 10^{-1}$ & $1.39 \times 10^{-1}[B]$ & $1.42 \times 10^{-1}$ & & $1.414 \times 10^{-1}$ & $1.414 \times 10^{-1}$ \\
\hline $1 s 3 p$ & $1 s 6 s$ & ${ }^{1} P^{\mathrm{o}}$ & ${ }^{1} S$ & 3 & 1 & $6.36 \times 10^{-3}$ & $9.75 \times 10^{-3}$ & $5.41 \times 10^{-3}[B]$ & $5.34 \times 10^{-3}$ & & & \\
\hline $1 s 3 p$ & $1 s 6 d$ & ${ }^{1} P^{\circ}$ & ${ }^{1} D$ & 3 & 5 & $5.67 \times 10^{-2}$ & $5.76 \times 10^{-2}$ & $5.53 \times 10^{-2}[B]$ & $5.64 \times 10^{-2}$ & & $5.623 \times 10^{-2}$ & \\
\hline
\end{tabular}

[15] Anderson and Weinhold.

[16] Kono and Hattori.

Estimated accuracy rates (Ref. [29]): $A^{\prime} \leq 2 \%, A \leq 3 \%, B^{\prime} \leq 7 \%, B \leq 10$. 
The lithium atom is the simplest system including core, valence, and valence-core interactions. $2 s-2 p$ transition has been a subject of controversy over years in lithium atom. The results given by Yan and Drake [4] and McAlexander et al. [5] are known to be very sensitive data. To illustrate the accuracy of our calculations, the absorption oscillator strengths are calculated for some multiplet dominant transitions and transitions between the low energy states in atomic lithium and are given in Table I. Our multiplet oscillator strength results have been compared with available data $[2,4,5,8,11]$ and accepted values taken from NIST [29]. The relevant parameters have been determined using both NCA wave functions and NRHF wave functions. Considering all transitions, our results calculated by using the parameters obtained from two different wave functions are in good agreement with data given in the literature. But especially for $2 s-2 p$ transition, the oscillator strength result calculated by using parameters determined from the NCA wave functions is in quite good agreement with available high-accuracy $a b$ initio data of Yan and Drake [4] and McAlexander et al. [5]. The average agreement of our oscillator strength results is within $0.6-0.7 \%$ to their results. This agreement indicates that the present results are reliable within confines of underlying the WBEPM theory.

The data in Table II present the oscillator strengths obtained by using the WBEPM theory for both multiplet and individual lines in $\mathrm{Li}(\mathrm{II})$ ion. The NRHF wave functions and the NCA wave functions are used for expectation values of radii in determination of relevant parameters. The data containing both multiplet and individual lines for comparisons in the literature are quite limited for many transitions which are considered in this study. Therefore, our results have been compared with available data [12, 15-17] only for multiplet values and with accepted values taken from NIST [29] and with atomic line data for both multiplet values and individual lines. The NIST values are given together with their accuracy rating in relevant columns. It can be shown from Table II that the oscillator strengths calculated by using parameters determined from NCA wave functions provide usually values in much better agreement with the results given in the literature for $\mathrm{Li}(\mathrm{II})$. The NRHF approximation is much more sophisticated than the numerical Coulomb approximation. The expectation values of the radii belonging to levels can be defined reliably by using the NRHF wave functions in many cases.

The results taken from NIST include the values of Wiese for dominant transitions and the values calculated from Coulomb approximation (CA) for other transitions belonging to excited levels. According to the CA method, the wave functions are constructed by using experimental binding energy of the active electron at large radial distances. If the active electron is in an inner orbital with very small amplitude outside the core or outer contribution to the matrix element belonging to the considering transition is very small, the assumptions used in the
CA method are not valid. Moreover, the major problem and error source of CA method is in approximating the atomic potential within the core region. To date, several attempts have been made to rectify this shortcoming, by assuming certain analytical or numerical approximations of the core part of the atomic potential [27, 30]. For example, in the NCA, the wave functions are obtained numerically by direct inward integration of Schrödinger's equation starting with the correct asymptotic boundary conditions. The integration is terminated at a certain small radial distance, so that the wave function is normalized to unity, and the obtained expectation value of radius agrees with the hydrogenic formula [27, 30].

The methods such as Coulomb approximation, quantum defect approximations and weakest bound electron potential model theory are known as semi-empirical methods. In all of these methods, the experimental binding energy of the active electron is used to construct an approximate radial wave function. While the results obtained from semi-empirical methods can be similar for large radial distances, these methods can present different results for small radial distances. The accuracy of the evaluated spectroscopic parameters such as oscillator strengths and transition probabilities depends upon the energies of initial and final levels and the ionization potential $E_{\text {limit }}$ of the atom in all of semi-empirical methods. However, the expectation values of radii belonging to levels play an important role in the WBEPM theory. According to this theory, the expectation values of radii belonging to levels can be calculated with known theoretical methods such as multiconfigurational Hartree-Fock (MCHF) method, numerical non-relativistic HartreeFock (NRHF), Roothaan-Hartree-Fock (RHF) method, Hartree-Kohn-Sham (HKS) method, time-dependent Hartree-Fock (TDHF), numerical Coulomb approximation (NCA) etc.

Previously, Celik et al. used expectation values of radii determined from NRHF wave functions and obtained very satisfactory transition probability results for atomic lithium [10] nitrogen [31, 32], oxygen [33], fluorine [34], sodium [35] and potassium [36]. The results obtained from this study clearly show that accurate expectation values of radii are necessary in the WBEPM theory framework. In this theory, the determination of $Z^{*}, n^{*}, l^{*}$ parameters is sufficient for the calculations of oscillator strength. The WBEPM theory can be used to calculate the oscillator strengths for both highly excited states and low lying states without any increase of complexity in calculation process.

\section{Acknowledgments}

This paper contains atomic line data obtained from the Atomic Line List v2.04, available at http://www.pa.uky.edu/ peter/atomic. The authors gratefully acknowledge the Selçuk University Scientific Research Projects (BAP) Coordinating Office for support. 


\section{References}

[1] T.C. Caves, A. Dalgarno, J. Quantum Spectrosc. Radiat. Transfer 12, 1539 (1972).

[2] E. Biemont, Astron. Astrophys. Suppl. Ser. 27, 489 (1977).

[3] R.N. Barnett, E.M. Johnson, W.A. Lester, Phys. Rev. A 51, 2049 (1995).

[4] Z.C. Yan, G.W.F. Drake, Phys. Rev. A 52, R4316 (1995).

[5] W.I. McAlexander, E.R.I. Abraham, N.W.M. Ritchie, C.J. Williams, H.T.C. Stoof, R.G. Hulet, Phys. Rev. A 51, R871 (1995).

[6] M.K. Chen, J. Phys. B, At. Mol. Opt. Phys. 29, 2179 (1996).

[7] L. Qu, Z. Wang, X. Guan, Chin. Phys. Lett. 14, 732 (1997).

[8] C.F. Fischer, M. Saparov, G. Gaigalas, M. Godafroid, At. Data Nucl. Data Tables 70, 119 (1998).

[9] L. Qu, Z. Wang, B. Li, Eur. Phys. J. D 5, 173 (1999).

[10] G. Çelik, J. Quantum Spectrosc. Radiat. Transfer 103, 578 (2007).

[11] J.Y. Zhang, J. Mitroy, M.V.J. Bromley, Phys. Rev. A 75, 0425091 (2007).

[12] N.M. Cann, A.J. Thakkar, Phys. Rev. A 46, 5397 (1992).

[13] B. Schiff, C.L. Pekeris, Y. Accad, Phys. Rev. A 4, 885 (1971).

[14] G.W.F. Drake, Phys. Rev. A 5, 614 (1972).

[15] M.T. Anderson, F. Weinhold, Phys. Rev. A 9, 118 (1974).

[16] A. Kono, S. Hattori, Phys. Rev. A 30, 2093 (1984).

[17] C.E. Theodosiou, Phys. Scr. 32, 129 (1985).

[18] R.D. Cowan, The Theory of Atomic Structure and Spectra, University of California Press, California 1981.
[19] I.I. Sobelman, Introduction to the Theory of Atomic Spectra, Pergamon Press, Oxford 1972.

[20] N.W. Zheng, Chin. Sci. Bull. 31, 1238 (1986).

[21] N.W. Zheng, A New Outline of Atomic Theory, Jiang su education Press, Nanjing 1988.

[22] N.W. Zheng, T. Wang, R. Yang, Y.G. Wu, J. Chem. Phys. 112, 7042 (2000).

[23] N.W. Zheng, Y.J. Sun, T. Wang, D.X. Ma, Y. Zhang, W. Su, Int. J. Quant. Chem. 76, 51 (2000).

[24] N.W. Zheng, T. Wang, R. Yang, J. Chem. Phys. 113, 6169 (2000)

[25] N.W. Zheng, Astr. J. Supp. Ser. 143, 231 (2002).

[26] N.W. Zheng, T. Wang, D.X. Ma, T. Zhou, J. Fan, Int. J. Quant. Chem. 98, 281 (2004).

[27] A. Lindgard, S.E. Nielsen, At. Data Nucl. Data Tabl. 19, 533 (1977).

[28] G. Gaigalas, C.F. Fischer, Comput. Phys. Commun 98, 255 (1996)

[29] Y. Ralchenko, F.C. Jou, D.E. Kelleher, A.E. Kramida, A. Musgrove, J. Reader, W.L. Wiese, K. Olsen, NIST Atomic Spectra Database (version 3.0.1) URL:http://physics.nist.gov, National Institute of Standards and Technology, Gaithersburg, MD 2008.

[30] C.E. Theodosiou, Phys. Rev. A 30, 2881 (1984).

[31] G. Celik, E. Akın, H.Ş. Kılıç, Eur. Phys. J. D 40, 325 (2006).

[32] G. Çelik, E. Akın, H.Ş. Kılıç, Int. J. Quant. Chem. 107, 495 (2007).

[33] G. Çelik, Ş. Ateş, Eur. Phys. J. D 44, 433 (2007).

[34] G. Çelik, H.Ş. Kılıç, E. Akın, T. J. Phys. 30, 165 (2006).

[35] G. Çelik, Ş. Ateş, Acta Phys. Pol. A 113, 1619 (2008).

[36] G. Çelik, Ş. Ateş, Can. J. Phys. 86, 487 (2008). 